\title{
Circadian Rhythm of Heart Rate Assessed by Wearable Devices Tends to Correlate with the Circadian Rhythm of Salivary Cortisol Concentration in Healthy Young Adults
}

\author{
Seunghwa Jeong ${ }^{1,2}$, Ju Yeon Seo ${ }^{1,2}$, Sehyun Jeon 1,2, Chul-Hyun Cho³, Ji Won Yeom¹, \\ Jaegwon Jeong ${ }^{1}$, Jung-Been Lee ${ }^{1,2}$, Taek Lee ${ }^{4}$, and Heon-Jeong Lee Le, $^{1,2}$ \\ ${ }^{1}$ Department of Psychiatry, Korea University College of Medicine, Seoul, Korea \\ ${ }^{2}$ Korea University Chronobiology Institute, Seoul, Korea \\ ${ }^{3}$ Department of Psychiatry, Chungnam National University Sejong Hospital, Sejong, Korea \\ ${ }^{4}$ Department of Convergence Security Engineering, College of Knowledge-based Services Engineering, Sungshin Women's University, Seoul, Korea
}

\begin{abstract}
Objective: The objective of this study was to determine whether the circadian rhythm of heart rate or step count using wearable devices was related to that of the salivary cortisol levels and to test the possibility that the data from wearable devices could be used as an indicator of circadian rhythm misalignment, which is emerging as a cause of insomnia and mood disorders. Methods: The heart rate and step count were continuously measured in 12 healthy young adults using wearable wrist devices for 5 days, and saliva was sampled every 4 hours, excluding sleeping time, for a total of 48 hours to measure the circadian rhythm of salivary cortisol concentration. Cortisol concentrations were assessed using the enzyme-linked immunosorbent assay. The cosinor analysis for the three measurements, salivary cortisol concentrations, heart rate, and step count, was used to estimate the circadian rhythm. Results: The mean values of the acrophase of the cosine-fitted curve of cortisol, heart rate, and step count were 9.06, 15.84, and 19.09, respectively, while those of the amplitude were 7.70, 12.60, and 10.68, respectively. In addition, the mean values of the mesor of the cosine-fitted curve for cortisol, heart rate, and step count were 17.19, 73.55 , and 45.45 , respectively, and those of robustness were $0.82,0.56$, and 0.18 , respectively. There was a possible positive correlation between the acrophase of the cosine-fitted curve of salivary cortisol and that of heart rate $(r=0.55, p=0.064)$. However, there was no correlation between the acrophase of the cosine-fitted curve of salivary cortisol and that of step count $(r=-0.2, p=0.533)$. Conclusion: The findings suggest that the heart rate measured using the wearable activity tracker was a relatively reliable biomarker of circadian rhythm.
\end{abstract}

Key Words: Circadian rhythm; Cortisol; Heart rate; Wearable activity tracker

Received: September 7, 2020 Revised: September 12, 2020 Accepted: September 13, 2020

Corresponding author: Heon-Jeong Lee, MD, PhD, Department of Psychiatry, Anam Hospital, Korea University College of Medicine, 73 Goryeodae-ro, Seongbukgu, Seoul 02841, Korea.

Tel: 82-2-920-6721, Fax: 82-2-927-9024, E-mail: leehjeong@korea.ac.kr

(a) This is an Open Access article distributed under the terms of the Creative Commons Attribution Non-Commercial License (https://creativecommons.org/licenses/by$\mathrm{nc} / 4.0)$ which permits unrestricted non-commercial use, distribution, and reproduction in any medium, provided the original work is properly cited.

\section{INTRODUCTION}

Due to living on Earth, which rotates on a 24-hour cycle, the human behavior and physiology has a circadian rhythm, which is a natural, internal process regulating the sleep-wake cycle and repeating itself approximately every 24 hours. It can refer to any biological process exhibiting an endogenous, entrainable oscillation of about 24 hours. Serum melatonin, cortisol, and core body temperature (CBT) rhythms are considered circadian biomarkers, controlled by a common circadian clock located in the hypothalamic suprachiasmatic nuclei $[1,2]$.

The human body circadian rhythm can be evaluated by repeatedly measuring CBT, or levels of melatonin and cortisol, throughout the day. CBT is a 24-hour rhythm that increases during the day and decreases at night, with the minimum temperature occurring at approximately $4 \mathrm{AM}$. These rhythm variations usually 
have amplitudes of more than $1.0^{\circ} \mathrm{C}$ [3]. Melatonin, the hormone produced by the pineal gland at night, serves as a time cue to the biological clock and promotes sleep anticipation in the brain. In most diurnal mammals, including humans, melatonin is secreted at night with a robust circadian rhythm and peak plasma levels occurring around 3 to $4 \mathrm{AM}$ [4]. Cortisol is a steroid hormone secreted by the adrenal glands, and its endogenous secretion is normally characterized by a robust circadian oscillation, with a daily peak in the early morning and minimal levels in the evening and early night [5].

The common methods of measuring human circadian rhythms are to repeatedly measure $\mathrm{CBT}$, melatonin concentration, or cortisol concentration, in a day. Repetitive hormone measurements, however, can be invasive and inconvenient and cannot be easily performed in everyday life. Repetitive measurements of CBT are also very cumbersome and laborious.

Therefore, the development of a simple, reliable method for circadian rhythm measurement will help evaluate circadian misalignment in clinical situations. We have considered a new approach to estimate endogenous circadian rhythms using wearable activity trackers, which were originally used to evaluate sleep variables, such as total sleep time, in-bed time, and sleep efficiency $[6,7]$. Several programs have been developed for the activity tracker, including those for measuring physical activity and biological rhythms [8] and circadian activity rhythms [9]. Various modern smart watches and wearable activity trackers feature an optical sensor that can estimate the wearer's heart rate. Heart rate monitoring has recently been reported to be a noninvasive and convenient method for estimating the circadian rhythm of CBT [10].

The purpose of this study was to determine whether the circadian rhythm of heart rate or step count measurements from wearable devices was related to that of the salivary cortisol concentration, and to test the possibility of using the circadian rhythm data from wearable devices as an indicator of circadian rhythm misalignment. This misalignment is emerging as a cause of many human illnesses, including insomnia, mood disorders, obesity, and cancers.

\section{METHODS}

\section{Participants}

A total of 16 young healthy subjects (11 males and 5 females) were assessed from August 30, 2017 to October 10, 2017. The ages of the participants (mean $\pm \mathrm{SD}$ ) were $29 \pm 3$ years. Through a structured diagnostic interview with a psychiatrist (H.-J. L) for major psychiatric disorders, according to the DSM-IV-TR (Diagnostic and Statistical Manual of Mental Disorders, 4th ed., Text-Revised) criteria [11] using the Korean version of the Mini International Neuropsychiatric Interview [12], the participants were confirmed to have no psychiatric illness. All participants underwent screening to exclude past or present major medical disorders, such as cardiovascular disease, metabolic disease (including diabetes mellitus), hormonal disease (including thyroid disease), and cancer. All par- ticipants completed questionnaires regarding their sleep conditions to exclude those with irregular or disturbed sleep/wake patterns. Among the 16 participants, four were excluded from the analysis because of their short device wear time.

All participants were informed of the purpose and procedures of the study, and all provided informed written consent prior to enrollment. The study protocol was approved by the Institutional Review Board of Korea University Anam Hospital (2017AN0279) and was conducted in accordance with the Declaration of Helsinki.

\section{Study procedures}

The Fitbit Charge 2 (Fitbit Inc., San Francisco, CA, USA) was used in the study. The data collected by the wearable device, such as the sleep, activity, and heart rate measurements, were automatically synchronized with a smartphone app using Bluetooth 4.0. Before the study, the settings of the wearable devices were matched. A Fitbit ID and password were created before the start of the study and provided to the participants for the synchronization of the information during the study period. We obtained raw data on sleep, activity, and heart rate at least five days during the study with the Fitbit application programming interface.

To analyze the circadian rhythms of cortisol, saliva was collected from the participants. Sample collection was performed at 08:00, 12:00, 16:00, 20:00, and 24:00 $\mathrm{h}$ for the last two consecutive days of the experiment. The participants provided saliva samples directly into Salivettes (Sarstedt AG \& Co., Nümbrecht, Germany) at each of the specified time points in the laboratory under staff supervision. The Salivette device was used according to the manufacturer's instructions and stored at $-80^{\circ} \mathrm{C}$ until the time of the assay. Cortisol levels of samples were checked by the enzyme-linked immunosorbent assay (ELISA) using Cortisol ELISA kits (ALPCO, Salem, NH, USA) according to the manufacturer's instructions.

\section{Cosinor fitting and data analyses}

Circadian outcome variables were derived from traditional parametric approaches such as cosinor model fitting [13]. Data were fitted with cosine curves using SigmaPlot software (Systat Software Inc., San Jose, CA, USA). The following circadian variables were extracted from the data: the mesor (the circadian rhythmadjusted mean values, which were based on the parameters of a cosine function), the amplitude (the difference between the peak and the mesor of a cosine function), and the acrophase (the time point at which the circadian peak occurred). Robustness was defined as a measure of statistical reliability and consistency of the model-fitted rhythm. The analysis of whether the cosine-fitted curve of heart rate or that of step count was related to the cosinefitted curve of salivary cortisol concentration was examined by the Pearson's correlation coefficient.

\section{RESULTS}

Figures 1 and 2 show the time plots of heart rate and step count, respectively, in 12 participants for 5 days. As shown in Figures 1 
and 2, the circadian rhythm of heart rate was more consistent than that of the step count in the cosine-fitted curve.

Table 1 shows the comparison of the mean values of the acro- phase, mesor, amplitude, and robustness of the cosine-fitted curves from the salivary cortisol concentration, heart rate, and step count from 12 participants. The mean values of the acrophase of the co-
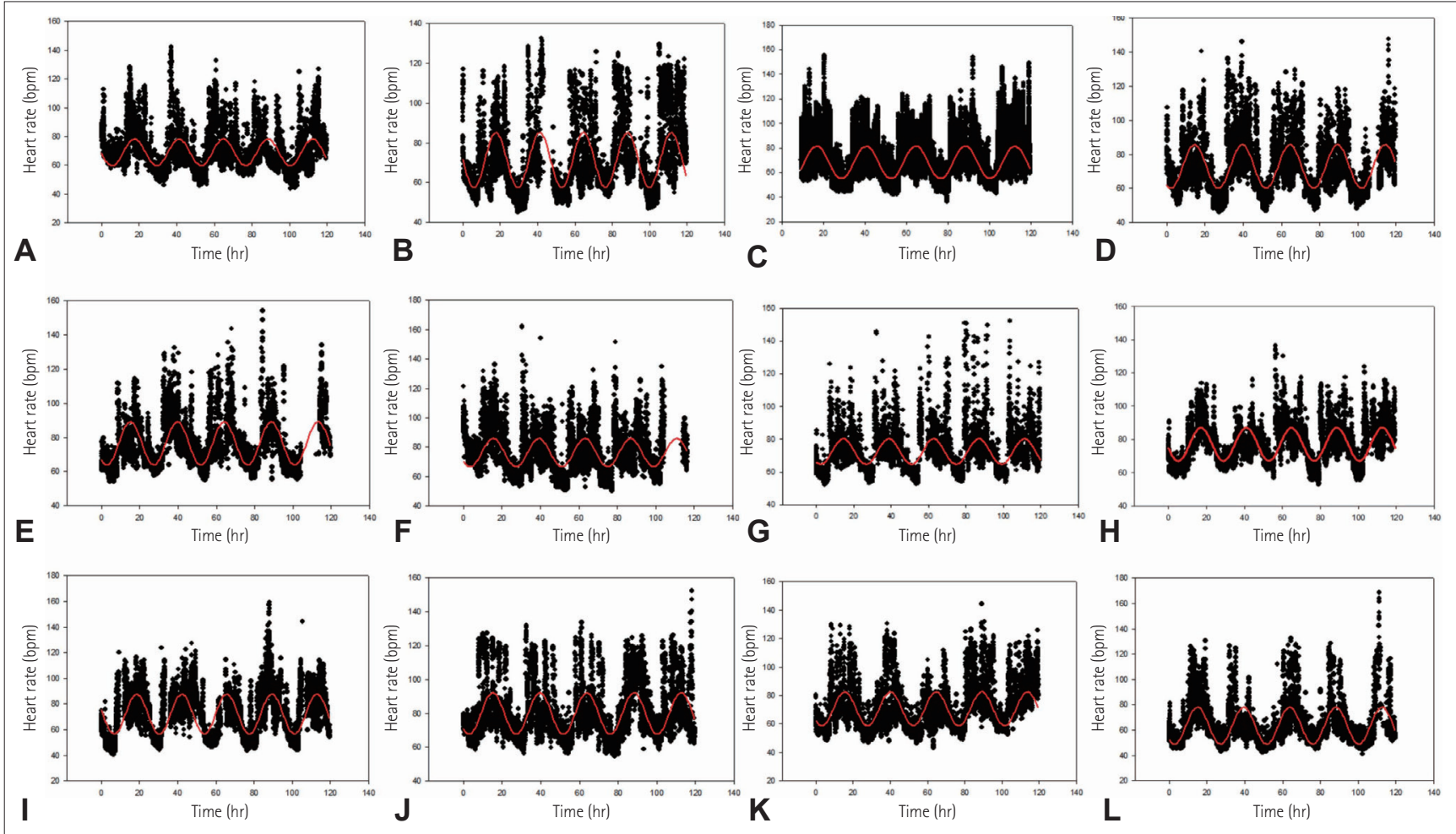

Figure 1. Time plots of the heart rate collected by the wearable device every minute for 5 consecutive days from 12 subjects (A-L). Curves in red show cosine-fitted curves from heart rate.
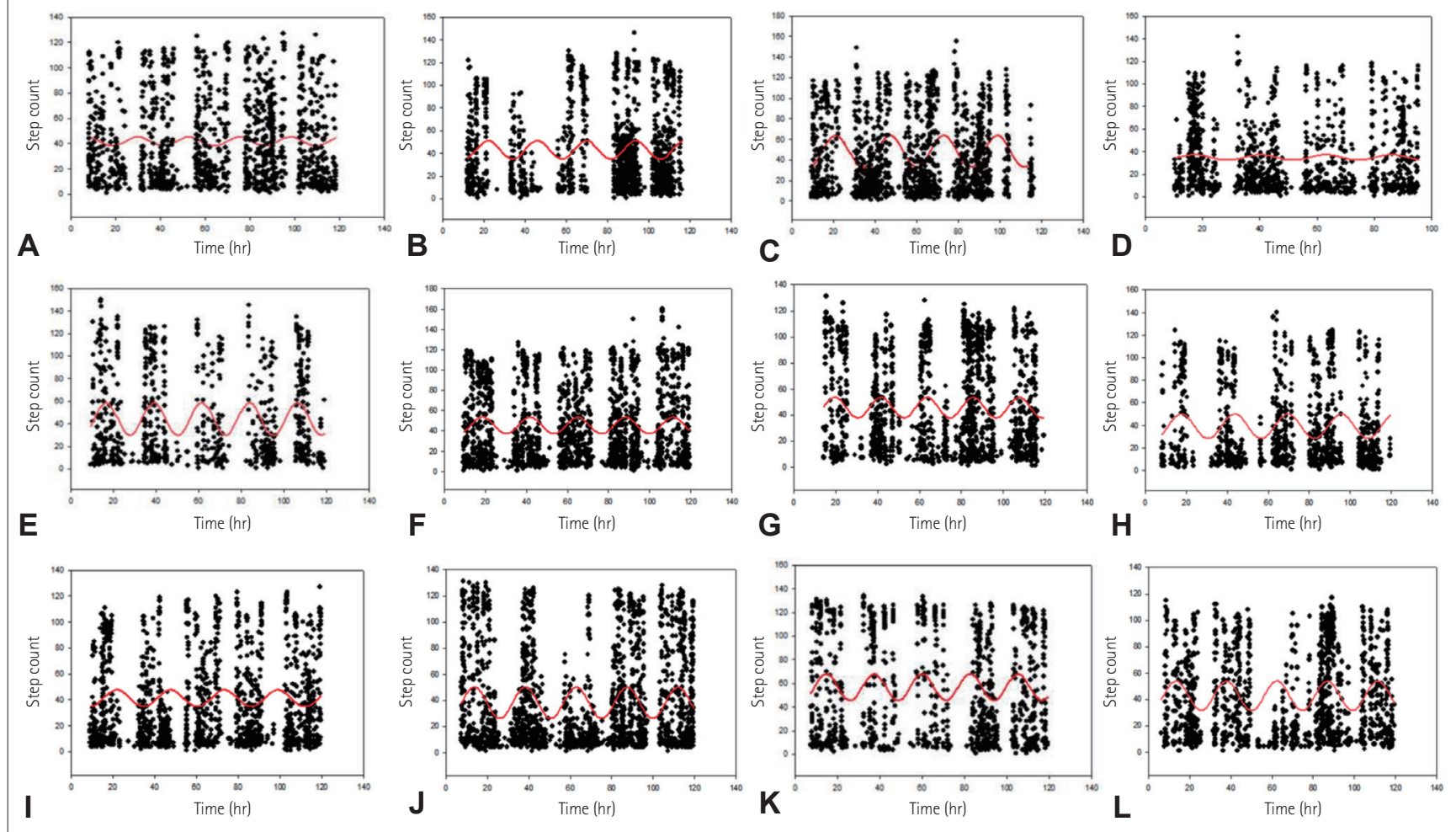

Figure 2. Time plots of the step count collected by the wearable device every minute for 5 consecutive days from 12 subjects (A-L). Curves in red show cosine-fitted curves from the step count. 
Table 1. Comparison of the mean values of acrophase, mesor, amplitude, and robustness of the cosine-fitted curves from the salivary cortisol concentration, heart rate, and step count

\begin{tabular}{lrrrr} 
& Acrophase & Mesor & Amplitude & Robustness \\
Salivary cortisol (2 days) & $9.06 \pm 1.01$ & $17.19 \pm 4.46$ & $7.70 \pm 1.59$ & $0.82 \pm 0.10$ \\
Heart rate (5 days) & $15.84 \pm 1.12$ & $73.55 \pm 4.49$ & $12.60 \pm 1.95$ & $0.56 \pm 0.07$ \\
Step count (5 days) & $19.09 \pm 3.12$ & $45.45 \pm 6.05$ & $10.68 \pm 2.34$ & $0.18 \pm 0.07$ \\
\hline
\end{tabular}

Data are presented as mean \pm standard deviation.

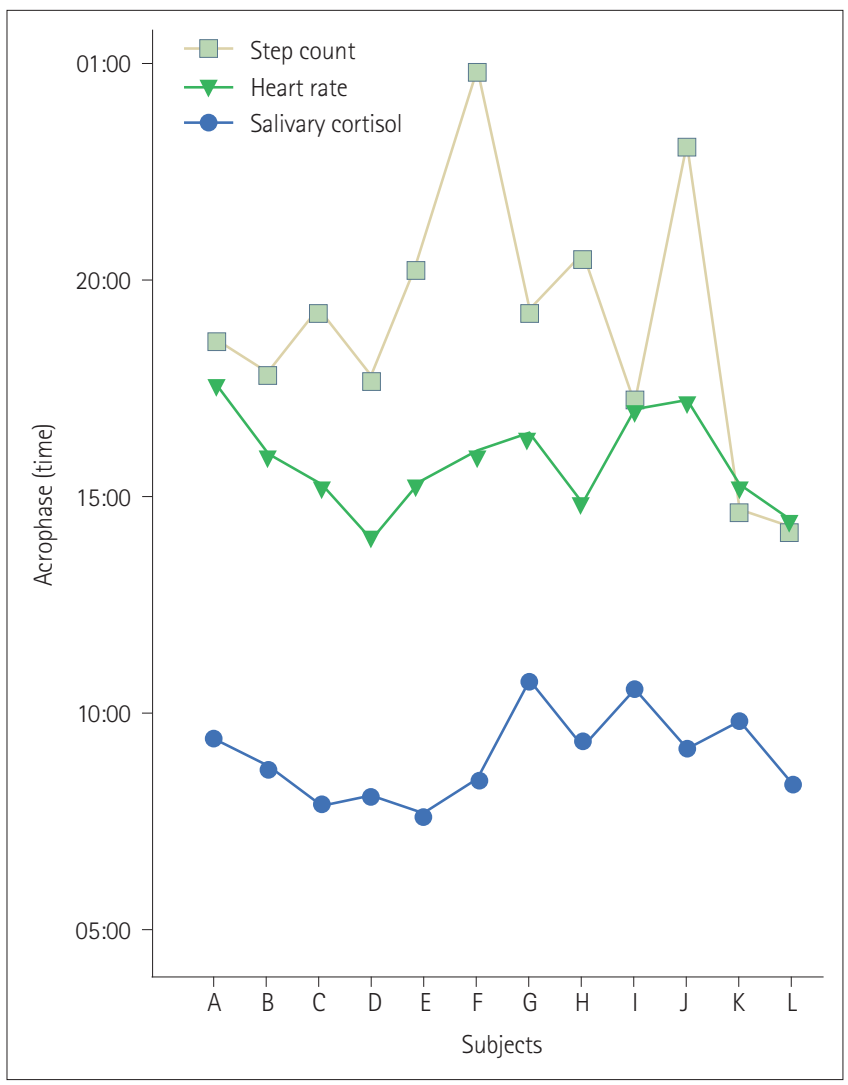

Figure 3. The comparison of the acrophases of cosine-fitted curves of heart rate, step count, and salivary cortisol concentration in 12 subjects.

sine-fitted curve of cortisol, heart rate, and step count were 9.06, 15.84 , and 19.09, respectively, while those of the amplitude were $7.70,12.60$, and 10.68 , respectively. In addition, the mean values of the mesor of the cosine-fitted curve of cortisol, heart rate, and step count were $17.19,73.55$, and 45.45 , respectively, and those of robustness were $0.82,0.56$, and 0.18 , respectively. Overall, among the cosine-fitted rhythm of the heart rate and step count obtained from the wearable device, the heart rate showed a more stable and reliable value. In particular, for robustness, which indicates statistical reliability and consistency of the model-fitted rhythm, salivary cortisol concentration and heart rate both had relatively high values of 0.56 and 0.82 , respectively, but the step count had a low value of 0.18 . Therefore, data of the salivary cortisol concentration and the heart rate were reliably cosine-curve fitted but that of step count was not.

Figure 3 shows acrophase of cosine-fitted curves of the 3 measurements in 12 participants: salivary cortisol concentration, heart rate, and step count. Although the time point of the acrophase var- ied, heart rate and salivary cortisol concentration showed similar patterns. However, step count showed a rather different pattern compared to the other two measurements. The correlation between the acrophase of cosine-fitted curve of heart rate and that of salivary cortisol concentration tended to be significantly correlated $(\mathrm{r}=0.551, \mathrm{p}=0.064)$. However, the acrophase of cosine-fitted curve of step count was not correlated with that of salivary cortisol ( $\mathrm{r}=$ $-0.2, \mathrm{p}=0.533$ ) (Figure 4).

\section{DISCUSSION}

Evidence for the association between circadian rhythm misalignment and several illnesses, such as insomnia, mood disorders, obesity, and cancers, is accumulating. Previously, our group reported circadian misalignment in hospitalized patients with bipolar disorders. The circadian rhythms in 31 episodes of bipolar disorders were evaluated at admission, at 2-week intervals during hospitalization, and at the time of discharge. Saliva and buccal cells were obtained at 8:00,11:00, 15:00, 19:00, and 23:00 h time points for two consecutive days and were used to analyze the circadian rhythm of cortisol and circadian rhythm of clock gene expression $[14,15]$. The study reported that acute manic episodes were associated with endogenous circadian rhythm acrophases averaging 7-hour in advance (earlier) than those of controls, although these acrophases could have resulted from an average of 17-hour clockwise delays. Mixed episodes were associated with 6-7-hour phase delays, whereas depression was associated with 4-5-hour phase delays compared to the controls. The phase shifts of these rhythms were normalized just before discharge. The study findings provided important evidence of circadian misalignment in mood disorders. However, measuring the salivary cortisol and circadian gene expression for the assessment of circadian rhythm was very complex and time-consuming and could not be used in clinical situations. Therefore, a simple and reliable method for circadian rhythm estimation should be developed to evaluate circadian misalignment of mood disorders in daily life and clinical settings.

In this study, the acrophase of the circadian rhythm of heart rate assessed using a wearable device tended to correlate with that of salivary cortisol concentration, while that of step count did not. This result suggests that the heart rate measured by the wearable device was a relatively more reliable biomarker for circadian rhythm than step count.

However, this study has some limitations. First, the sample size was too small to make conclusions; the association between cir- 

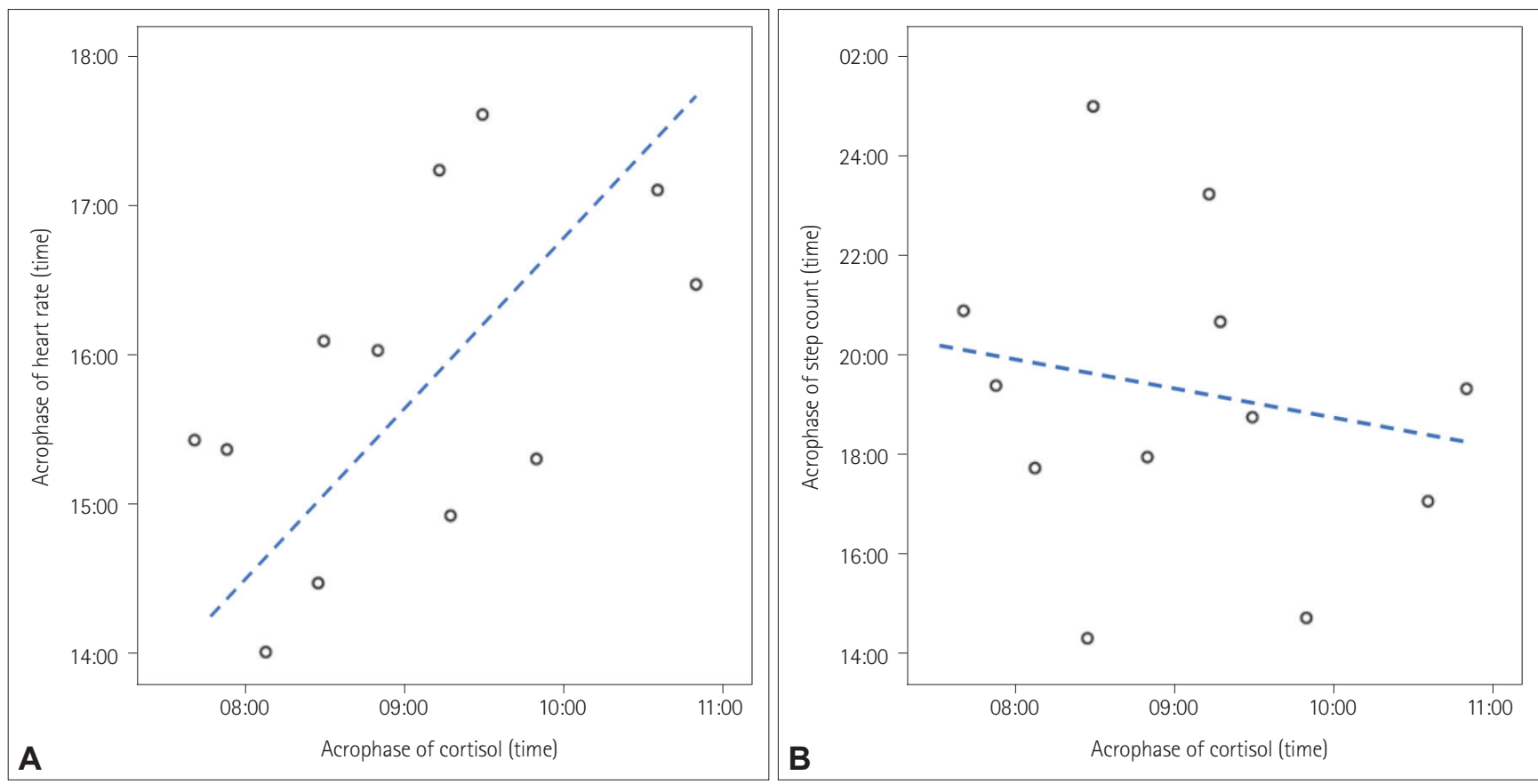

Figure 4. Scatterplots show the correlation between the acrophase of the cosine-fitted curve of heart rate and that of salivary cortisol concentration $(r=0.551, p=0.064)(A)$ and the correlation between the acrophase of the cosine-fitted curve of step count and that of salivary cortisol concentration $(r=-0.2, p=0.533)(B)$.

cadian rhythms in the heart rate and those in the salivary cortisol did not reach statistical significance. Second, although the wearable devices were worn for five days, saliva sampling for cortisol concentration was performed for only two days. Therefore, there may be some limitations in comparing them. Nevertheless, automatic measurement of heart rate using wearable devices would be a relatively simple method compared to conventional measurement methods of endogenous circadian rhythm, which include repeated measurements of more than 24 hours of CBT, melatonin concentration, and cortisol concentration. Taking these limitations into account, further investigation is needed, including larger sample sizes and longer duration studies, to verify the reliability of the circadian rhythm assessment based on the heart rate measured by the wearable device.

\section{Acknowledgments}

This study was supported by the Korea Health 21 R\&D Project funded by the National Research Foundation of Korea (2017M3A9F1031220 and 2019R1A2C2084158).

\section{Conflicts of Interest}

The authors have no potential conflicts of interest to disclose.

\section{Author Contributions}

Conceptualization: Heon-Jeong Lee. Formal analysis: HeonJeong Lee, Seunghwa Jeong, Jung-Been Lee, Taek Lee. Funding acquisition: Heon-Jeong Lee. Investigation: Heon-Jeong Lee, Seunghwa Jeong, Ju Yeon Seo, Chul-Hyun Cho, Ji Won Yeom, Jaegwon Jeong. Methodology: Heon-Jeong Lee, Chul-Hyun Cho. Project administration: Heon-Jeong Lee. Resources: Heon-Jeong
Lee. Supervision: Heon-Jeong Lee. Software: Seunghwa Jeong, Heon-Jeong Lee, Jung-Been Lee, Taek Lee. Validation: HeonJeong Lee, Chul-Hyun Cho, Sehyun Jeon. Visualization: HeonJeong Lee, Seunghwa Jeong. Writing_original draft: Seunghwa Jeong, Heon-Jeong Lee. Writing_-review \& editing: Heon-Jeong Lee, Seunghwa Jeong.

\section{ORCID iDs}

Heon-Jeong Lee (1)

https://orcid.org/0000-0002-9560-2383

Seunghwa Jeong (i)

https://orcid.org/0000-0003-3850-9243

\section{REFERENCES}

1. Pina G, Brun J, Tissot S, Claustrat B. Long-term alteration of daily melatonin, 6-sulfatoxymelatonin, cortisol, and temperature profiles in burn patients: a preliminary report. Chronobiol Int 2010;27:378-392.

2. Verceles AC, Silhan L, Terrin M, Netzer G, Shanholtz C, Scharf SM. Circadian rhythm disruption in severe sepsis: the effect of ambient light on urinary 6-sulfatoxymelatonin secretion. Intensive Care Med 2012;38:804-810.

3. Lack LC, Gradisar M, Van Someren EJ, Wright HR, Lushington K. The relationship between insomnia and body temperatures. Sleep Med Rev 2008; 12:307-317.

4. Zisapel N. New perspectives on the role of melatonin in human sleep, circadian rhythms and their regulation. Br J Pharmacol 2018;175:3190-3199.

5. Oster H, Challet E, Ott V, Arvat E, de Kloet ER, Dijk DJ, et al. The functional and clinical significance of the 24-hour rhythm of circulating glucocorticoids. Endocr Rev 2017;38:3-45.

6. Kripke DF, Mullaney DJ, Messin S, Wyborney VG. Wrist actigraphic measures of sleep and rhythms. Electroencephalogr Clin Neurophysiol 1978;44: 674-676.

7. Mullaney DJ, Kripke DF, Messin S. Wrist-actigraphic estimation of sleep time. Sleep 1980;3:83-92.

8. White M, Kiel K, Alexander D, Powell G. Computer-compatible activity 
measurement of children: wrist actigraph standardization. Comput Nurs 1984;2:125-129.

9. Brown AC, Smolensky MH, D’Alonzo GE, Redman DP. Actigraphy: a means of assessing circadian patterns in human activity. Chronobiol Int 1990;7:125-133.

10. Sim SY, Joo KM, Kim HB, Jang S, Kim B, Hong S, et al. Estimation of circadian body temperature rhythm based on heart rate in healthy, ambulatory subjects. IEEE J Biomed Health Inform 2017;21:407-415.

11. American Psychiatric Association. Diagnostic and statistical manual of mental disorders, fourth edition, text revision. Washington, DC: American Psychiatric Publishing, 2000.

12. Yoo SW, Kim YS, Noh JS, Oh KS, Kim CH, Namkoong K, et al. Validity of
Korean version of the Mini-International Neuropsychiatric Interview. Anxiety Mood 2006;2:50-55.

13. Refinetti R, Lissen GC, Halberg F. Procedures for numerical analysis of circadian rhythms. Biol Rhythm Res 2007;38:275-325.

14. Moon JH, Cho CH, Son GH, Geum D, Chung S, Kim H, et al. Advanced circadian phase in mania and delayed circadian phase in mixed mania and depression returned to normal after treatment of bipolar disorder. EBioMedicine 2016;11:285-295.

15. Cho CH, Moon JH, Yoon HK, Kang SG, Geum D, Son GH, et al. Molecular circadian rhythm shift due to bright light exposure before bedtime is related to subthreshold bipolarity. Sci Rep 2016;6:31846. 